\title{
XPD Lys751Gln and Asp312Asn Polymorphisms and Susceptibility to Skin Cancer: A Meta-Analysis of 17 Case- control Studies
}

\author{
Hai-Li Zhu ${ }^{1,2 \&}$, Ji-Ming Bao ${ }^{3 \&}$, Pei-Xin Lin ${ }^{1,2}$, Wen-Xia Li ${ }^{1,2}$, Zhen-Ning Zou ${ }^{1,2}$, \\ Ye-En Huang ${ }^{1,2}$, Qing Chen ${ }^{4}$, Hong Shen ${ }^{1,2 *}$
}

\begin{abstract}
Background: Numerous studies have explored the influence of XPD Lys751GIn and/or Asp312Asn polymorphisms on skin cancer susceptibility. However, the results remain inconclusive. To derive a more precise estimation, we conducted a comprehensive search to identify all available published studies and performed a meta-analysis. Materials and Methods: Electronic literature searches of the PubMed, CBM and CNKI databases were performed up to March 2014. Odds ratios (ORs) with 95\% confidence intervals (CIs) were applied to assess the strength of associations. Results: Seventeen case-control studies were included with a total sample size of 6, 113 cases and 11, 074 controls for the XPD Lys751Gln polymorphism, and 10 studies $(3,840$ cases and 7, 637 controls) for the XPD Asp312Asn polymorphism were pooled for analysis. Overall, no significant associations were found between the XPD Lys751GIn polymorphism and skin cancer risk in any genetic model. On stratified analysis by tumor type, XPD Lys751GIn polymorphism was not associated with increased risk of non-melanoma skin cancer, but was significantly related with increased risk of cutaneous melanoma (Gln/Gln $v s$ Lys/Lys: OR=1.15, 95\% CI=1.02-1.29, $p=0.023$; dominant model: $\mathrm{OR}=1.09,95 \% \mathrm{CI}=1.01-1.18, p=0.036$ ). For the XPD Asp312Asn polymorphism, no significant association with skin cancer risk was observed in overall or subgroup analyses. Conclusions: The present meta-analysis suggests that the XPD Lys751Gln polymorphism may contribute to the risk of cutaneous melanoma from currently available evidence. Further investigations are needed to obtain more insight into possible roles of these two polymorphisms in skin carcinogenesis.
\end{abstract}

Keywords: XPD - Lys751Gln - Asp312Asn - polymorphisms - skin cancer - meta-analysis

Asian Pac J Cancer Prev, 15 (16), 6619-6625

\section{Introduction}

Skin cancer, one of the most common neoplasms, has been an important public health problem in most Caucasian populations with the increasing incidence during the last few years (Leiter and Garbe, 2008). Statistics show that more than two million cases of skin cancer are diagnosed in American every year (Siegel et al., 2012). According to histological types, skin cancer can be subdivided into cutaneous melanoma, squamous cell carcinoma (SCC) and basal cell carcinoma (BCC), respectively (Rubin et al., 2005; Thompson et al., 2005).

Skin cancer has a complex etiology and pathogenesis caused by the interaction of environmental and inherited factors (Ji et al., 2012). It is widely accepted that inappropriate exposure to ultraviolet radiation (UVR) which causes various kinds of DNA damage, is critical in the occurrence and development of skin cancer (Armstrong et al., 2001). In addition, extensive epidemiological evidence has revealed that the incidence of skin cancer is variable in noncancer subjects with different ethnic backgrounds, suggesting host genetic susceptibility plays an important role in skin cancer risk among different ethnicities (Capell et al., 2009; Lin et al., 2011).

Xeroderma pigmentosum group D (XPD), also named excision repair cross-complementing rodent repair deficiency Group 2 (ERCC2), is one of the most important DNA repair genes. It plays a vital role in nucleotide excision repair (NER) pathway, which removes bulky adducts, such as those caused by environmental agents, UVR induced DNA damage, crosslinks and oxidative damage (Sancar et al., 1993; Weeda et al., 1993). Several important single-nucleotide polymorphisms (SNPs) have been identified in the XPD locus. Among them, Lys751Gln and Asp312Asn polymorphisms were most extensively investigated. The XPD Lys751Gln polymorphism (rs13181) in codon 751 of exon 23, an A to C substitution, results in a Lys to Gln amino acid exchange (Shen et al., 1998; Pabalan et al., 2010). And Asp312Asn polymorphism (rs1799793), a G to A substitution in codon

${ }^{1}$ Department of Pathology, Nanfang Hospital, Southern Medical University, ${ }^{2}$ Department of Pathology, School of Basic Medical Sciences, Southern Medical University, ${ }^{3}$ Department of Urology, Nanfang Hospital, Southern Medical University, ${ }^{4}$ Department of Epidemiology, School of Public Health and Tropical Medicine, Southern Medical University, Guangzhou, China ${ }^{\star}$ Equal contributors *For correspondence: shenhong2010168@163.com. 
312 of exon 10, produces Asp to Asn amino acid exchange (Shen et al., 1998; Pabalan et al., 2010).

To date, plenty of studies have explored the associations of XPD Lys751Gln and/or Asp312Asn polymorphisms with skin cancer susceptibility. Nevertheless, findings on the direction of the associations remain equivocal. To derive a more precise estimation, we conducted a comprehensive search to identify all available published studies and performed this meta-analysis.

\section{Materials and Methods}

\section{Identification and eligibility of studies}

Eligible articles up to March 2014 were identified by searching the electronic literatures of PubMed, CBM (Chinese Biomedical) and CNKI (China National Knowledge Infrastructure) databases. The search terms were used as follows: "xeroderma pigmentosum group D", "XPD”, "ERCC2", “cutaneous melanoma”, "basal cell carcinoma", "squamous cell carcinoma of skin" and combined phrases. There were not any restrictions on sample size, population, or language. All eligible studies were retrieved, and their bibliographies were checked as well for other relevant publications. Studies were required to comply with the following inclusion criteria: (1) case-control studies; (2) the studies should evaluate the association of XPD Lys751Gln and/or Asp312Asn polymorphisms with risk of skin cancer; (3) sufficient information to calculate odds ratios (ORs) with $95 \%$ confidence intervals (CIs); (4) For multiple publications reporting on overlapping data, only the most recent, largest or complete study was selected; and (5) the distributions of genotypes among controls conformed to the HardyWeinberg equilibrium (HWE) ( Salanti et al., 2005).

\section{Data extraction}

Data of eligible studies were carefully extracted by two independent authors (HZ and JB) according to prespecified selection criteria. A consensus meeting was then held to resolve any discrepancies. The following information was collected: name of the first author, publication year, ethnicity, country, number of cases and controls, distributions of every genotype, tumor type and $\mathrm{P}$ value for Hardy-Weinberg equilibrium (HWE).

\section{Statistical analysis}

All statistical analyses were performed using Stata statistical software, version 12.0 (Stata Corp, College Station, TX, USA). The chi-square goodness of fit was used to test deviation from HWE, statistical significance was defined as $p<0.05$.

The crude odds ratios (ORs), as well as their $95 \%$ confidence intervals (95\% CIs) were calculated to assess the strength of associations between XPD Lys751Gln and/ or Asp312Asn polymorphisms and skin cancer risk. The pooled ORs were performed with codominant model (Lys/ Gln $v s$ Lys/Lys and Asp/Asn vs Asp/Asp; Gln/Gln vs Lys/ Lys and Asn/Asn vs Asp/Asp), dominant model (Lys/Gln + Gln/Gln vs Lys/Lys and Asp/Asn + Asn/Asn vs Asp/Asp) and recessive model (Gln/Gln vs Lys/Gln + Lys/Lys and Asn/Asn vs Asp/Asn + Asp/Asp), respectively.
Heterogeneity among the studies in terms of degree of association was assessed using $\chi^{2}$ tests. The $\mathrm{I}^{2}$ statistic was used to estimate the percentage of variation between the results that was caused by heterogeneity, rather than sampling error $\left(p<0.10\right.$ or $\mathrm{I}^{2}>50 \%$ was treated as significant heterogeneity). When heterogeneity was detected, the random-effects model (DerSimonianLaird method) (DerSimonian et al., 1986) was used to calculate the pooled ORs; otherwise, the fixed-effects model (Mantel-Haenszel method) was selected (Mantel et al., 1959). To evaluate the tumor type-specific effects, subgroup analysis was performed by tumor type. Sensitivity analyses were performed to see whether any exclusion of the studies could affect the initial results.

Moreover, potential publication bias was observed and evaluated by visual inspection of the Begg's funnel plots. We also employed the Begg's adjusted correlation test (Begg et al., 1994) to evaluate the possible publication bias $(p<0.05$ was considered representative of statistically significant publication bias).

\section{Results}

\section{Study selection and characteristics}

Based on the search terms, 58 individual literatures were initially found in the selected databases. After screening the titles and abstracts, only 20 potentially eligible articles were identified for further detailed evaluation. After full-text assessment of these articles, we included a total of 15 articles (Dybdahl et al., 1999; Winsey et al., 2000; Vogel et al., 2001; Yin et al., 2003; Baccarelli et al., 2004; Lovatt et al., 2005; Festa et al., 2005; Han et al., 2005; Thirumaran et al., 2006; Li et al., 2006; Millikan et al., 2006; Debniak et al., 2006; Povey et al., 2007; Kertat et al., 2008; Paszkowska-Szczur et al., 2013) in this meta analysis, including 9 studies for cutaneous melanoma (Winsey et al., 2000; Baccarelli et al., 2004; Han et al., 2005; Li et al., 2006; Millikan et al., 2006; Debniak et al., 2006; Povey et al., 2007; Kertat et al., 2008; Paszkowska-Szczur et al., 2013), 7 studies for basal cell carcinoma (Dybdahl et al., 1999; Vogel et al., 2001; Yin et al., 2003; Lovatt et al., 2005; Festa et al., 2005; Han et al., 2005; Thirumaran et al., 2006) and 1 for squamous cell carcinoma (Han et al., 2005). All articles were written in English. A flow diagram of the search process was shown in Figure 1.

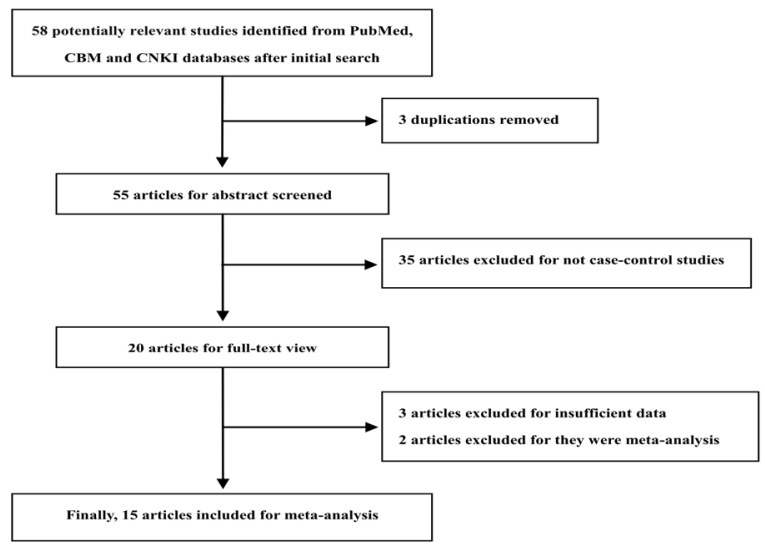

Figure 1. Flow Diagram of the Study Selection Process 

Table 1. Characteristics of the Studies of XPD Lys751Gln Polymorphism and Susceptibility to Skin Cancer

\begin{tabular}{|c|c|c|c|c|c|c|c|c|c|c|c|c|}
\hline \multirow[t]{3}{*}{ First auther } & \multirow[t]{3}{*}{ Ethnicity } & \multirow[t]{3}{*}{ Country } & \multirow{2}{*}{\multicolumn{2}{|c|}{ Sample Size }} & \multicolumn{5}{|c|}{ Genotype Distributions } & \multirow{3}{*}{\multicolumn{2}{|c|}{ Tumor type }} & \multirow[t]{3}{*}{$P_{H W E}$} \\
\hline & & & & & \multicolumn{3}{|c|}{ Case } & \multicolumn{2}{|r|}{ Control } & & & \\
\hline & & & Case & Control & Lys/Lys & Lys/ Gln & Gln/Gln & Lys/Lys & Lys/ Gln & & & \\
\hline Dybdahl (1999) & Caucasian & Denmark & 40 & 40 & 21 & 17 & 2 & 17 & 16 & 7 & $\mathrm{BCC}$ & 0.65 \\
\hline Winsey (2000) & Caucasian & UK & 125 & 211 & 47 & 54 & 24 & 72 & 107 & 32 & melanoma & 0.75 \\
\hline Vogel (2001) & Caucasian & Denmark & 71 & 117 & 24 & 35 & 12 & 44 & 61 & 12 & $\mathrm{BCC}$ & 0.39 \\
\hline Yin (2003) & Caucasian & Denmark & 20 & 20 & 10 & 9 & 1 & 8 & 8 & 4 & $\mathrm{BCC}$ & 0.86 \\
\hline Baccarelli (2004) & Caucasian & Italy & 176 & 177 & 58 & 94 & 24 & 59 & 85 & 33 & melanoma & 0.92 \\
\hline Han (2005a) & Caucasian & USA & 203 & 844 & 81 & 99 & 23 & 295 & 415 & 134 & melanoma & 0.84 \\
\hline Han (2005b) & Caucasian & USA & 286 & 844 & 98 & 141 & 47 & 295 & 415 & 134 & $\mathrm{BCC}$ & 0.84 \\
\hline Han (2005c) & Caucasian & USA & 280 & 844 & 126 & 112 & 42 & 295 & 415 & 134 & $\mathrm{SCC}$ & 0.84 \\
\hline Lovatt (2005) & Caucasian & UK & 509 & 379 & 217 & 218 & 74 & 149 & 177 & 53 & $\mathrm{BCC}$ & 0.99 \\
\hline Festa (2005) & Caucasian & Sweden & 197 & 561 & 69 & 94 & 34 & 194 & 282 & 85 & $\mathrm{BCC}$ & 0.57 \\
\hline Li (2006) & Caucasian & USA & 602 & 603 & 219 & 297 & 86 & 255 & 270 & 78 & melanoma & 0.89 \\
\hline Millikan (2006) & Caucasian & USA & 1212 & 2436 & 441 & 576 & 195 & 981 & 1128 & 327 & melanoma & 0.99 \\
\hline Debniak (2006) & Caucasian & Poland & 426 & 1141 & 146 & 207 & 73 & 432 & 547 & 162 & melanoma & 0.87 \\
\hline Thirumaran (2006) & Caucasian & Hungary & 529 & 533 & 174 & 269 & 86 & 179 & 262 & 92 & $\mathrm{BCC}$ & 0.97 \\
\hline Povey (2007) & Caucasian & UK & 507 & 438 & 242 & 200 & 65 & 206 & 177 & 55 & melanoma & 0.22 \\
\hline Kertat (2008) & Caucasian & Sweden & 241 & 251 & 78 & 122 & 41 & 82 & 135 & 34 & melanoma & 0.18 \\
\hline Paszkowska-Szczur (2013) & Caucasian & Poland & 689 & 1635 & 245 & 325 & 119 & 592 & 767 & 276 & melanoma & 0.59 \\
\hline
\end{tabular}

BCC, basal cell carcinoma; SCC, squamous cell carcinoma; PHWE, $P$ value of Hardy -Weinberg equilibrium

Table 2. Characteristics of the Studies of XPD Asp312Asn Polymorphism and Susceptibility to Skin Cancer

\begin{tabular}{|c|c|c|c|c|c|c|c|c|c|c|c|c|}
\hline \multirow[t]{3}{*}{ First auther } & \multirow[t]{3}{*}{ Ethnicity } & \multirow[t]{3}{*}{ Country } & \multirow{2}{*}{\multicolumn{2}{|c|}{ Sample Size }} & & \multicolumn{4}{|c|}{ Genotype Distributions } & \multirow{3}{*}{\multicolumn{2}{|c|}{ Tumor type }} & \multirow[t]{3}{*}{$P_{H W E}$} \\
\hline & & & & & \multicolumn{3}{|c|}{ Case } & \multicolumn{2}{|r|}{ Control } & & & \\
\hline & & & Case & Control & Asp/Asp & Asp/Asn & Asn/Asn & Asp/Asp & Asp/Asn & & & \\
\hline Winsey (2000) & Caucasian & UK & 125 & 211 & 49 & 54 & 22 & 89 & 95 & 27 & melanoma & 0.98 \\
\hline Vogel (2001) & Caucasian & Denmark & 68 & 105 & 29 & 25 & 14 & 46 & 39 & 20 & $\mathrm{BCC}$ & 0.1 \\
\hline Baccarelli (2004) & Caucasian & Italy & 164 & 172 & 52 & 94 & 18 & 59 & 89 & 24 & melanoma & 0.58 \\
\hline Han (2005a) & Caucasian & USA & 206 & 836 & 88 & 99 & 19 & 342 & 373 & 121 & melanoma & 0.5 \\
\hline Han (2005b) & Caucasian & USA & 285 & 836 & 104 & 149 & 32 & 342 & 373 & 121 & $\mathrm{BCC}$ & 0.5 \\
\hline $\operatorname{Han}(2005 c)$ & Caucasian & USA & 280 & 836 & 128 & 115 & 37 & 342 & 373 & 121 & SCC & 0.5 \\
\hline Lovatt (2005) & Caucasian & UK & 509 & 379 & 224 & 219 & 66 & 151 & 163 & 65 & $\mathrm{BCC}$ & 0.19 \\
\hline Debniak (2006) & Caucasian & Poland & 425 & 1262 & 168 & 188 & 69 & 492 & 597 & 173 & melanoma & 0.93 \\
\hline Li (2006) & Caucasian & USA & 602 & 603 & 242 & 290 & 70 & 273 & 259 & 71 & melanoma & 0.73 \\
\hline Millikan (2006) & Caucasian & USA & 1176 & 2397 & 1039 & 1098 & 162 & 1039 & 1098 & 260 & melanoma & 0.49 \\
\hline
\end{tabular}

BCC, basal cell carcinoma; SCC, squamous cell carcinoma; PHWE, $P$ value of Hardy -Weinberg equilibrium

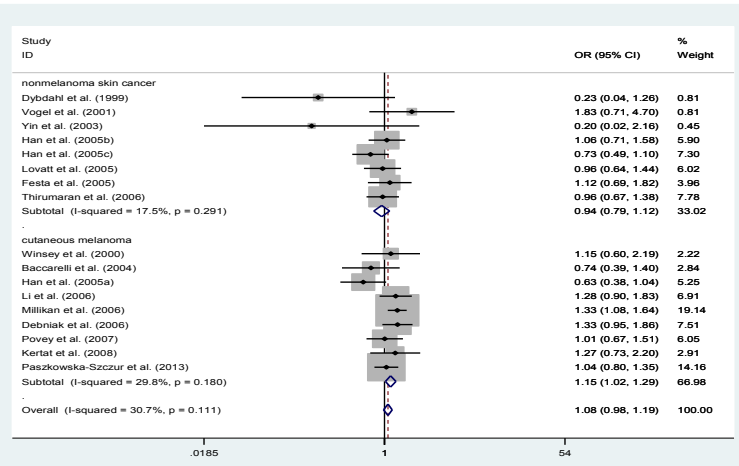

Figure 2. Meta-Analysis of the Association between XPD Lys751GIn Polymorphism and Skin Cancer (Asn/ Asn vs Asp/Asp)

Concerning XPD Lys751Gln polymorphisms, 17 studies of the included 15 articles were eligible with a total sample size of 6113 cases and 11074 controls. With respect to XPD Asp312Asn polymorphism, 10 studies were pooled for analysis (3840 cases and 7637 controls). The characteristics of included studies were presented in Table 1 and Table 2.

Meta-analysis results

For all included studies, the allelic distributions of Lys751Gln and Asp312Asn in the control group were

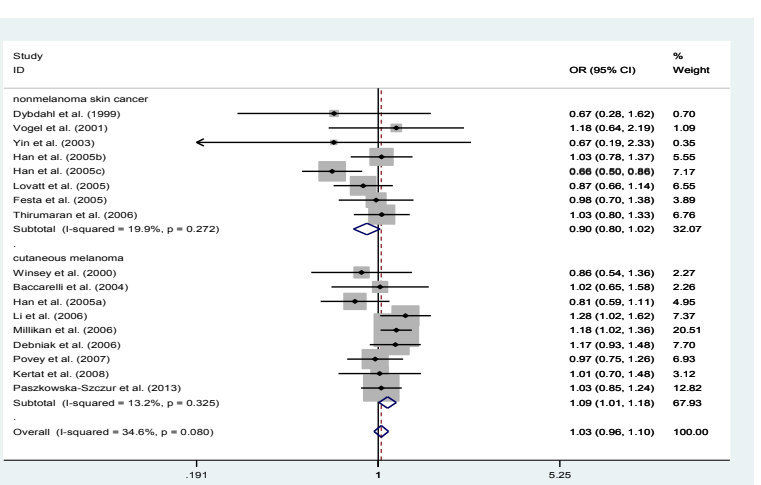

Figure 3. Meta-Analysis of the Association between XPD Lys751Gln Polymorphism and Skin Cancer (Dominant Model)

consistent with HWE at the 0.05 level (Table 1-2), indicating that obvious effects of natural selection and migration on genetic equilibrium had been avoided.

The association between XPD Lys751Gln polymorphism and skin cancer was shown in Table 3. The heterogeneity for all studies was only significant in dominant model $\left(\mathrm{I}^{2}=34.6, p=0.080\right)$, so the randomeffects model was used. The fixed-effects model was performed in other genetic models. No association was observed between the polymorphism and skin cancer for overall population at all genetic contrasts (Lys/ Gln 
Table 3. Meta-analysis of the XPD Lys751Gln Polymorphism with Risk of Skin Cancer

\begin{tabular}{llrlllllcc}
\hline Comparison & Tumor type & $\mathrm{N}$ & $\mathrm{OR}$ & $95 \% \mathrm{CI}$ & $P^{a}$ & Model & $\chi^{2}$ & $P^{b}$ & $\mathrm{I}^{2}$ \\
\hline Lys/ Gln vs. Lys/Lys & Overall & 17 & 1.01 & $0.94-1.09$ & 0.71 & $\mathrm{~F}$ & 0.0067 & 0.194 & 22.3 \\
& melanoma & 9 & 1.07 & $0.98-1.16$ & 0.113 & $\mathrm{~F}$ & 0 & 0.517 & 0 \\
& NMSC & 8 & 0.89 & $0.78-1.01$ & 0.082 & $\mathrm{~F}$ & 0.0054 & 0.33 & 12.8 \\
& BCC & 7 & 0.97 & $0.84-1.12$ & 0.648 & $\mathrm{~F}$ & 0 & 0.956 & 0 \\
Gln/Gln vs. Lys/Lys & Overall & 17 & 1.08 & $0.98-1.19$ & 0.133 & $\mathrm{~F}$ & 0.0198 & 0.111 & 30.7 \\
& melanoma & 9 & 1.15 & $1.02-1.29$ & 0.023 & $\mathrm{~F}$ & 0.0149 & 0.18 & 29.8 \\
& NMSC & 8 & 0.94 & $0.79-1.12$ & 0.493 & $\mathrm{~F}$ & 0.015 & 0.291 & 17.5 \\
dominant model & BCC & 7 & 1 & $0.82-1.22$ & 0.991 & $\mathrm{~F}$ & 0.0078 & 0.36 & 9 \\
& Overall & 17 & 1 & $0.92-1.10$ & 0.957 & $\mathrm{R}$ & 0.0109 & 0.08 & 34.6 \\
& melanoma & 9 & 1.09 & $1.01-1.18$ & 0.036 & $\mathrm{~F}$ & 0.0024 & 0.325 & 13.2 \\
recessive model & NMSC & 8 & 0.9 & $0.80-1.02$ & 0.094 & $\mathrm{~F}$ & 0.0081 & 0.272 & 19.9 \\
& BCC & 7 & 0.97 & $0.85-1.11$ & 0.686 & $\mathrm{~F}$ & 0 & 0.878 & 0 \\
& Overall & 17 & 1.07 & $0.98-1.17$ & 0.134 & $\mathrm{~F}$ & 0.0052 & 0.312 & 12.2 \\
& melanoma & 9 & 1.1 & $0.99-1.23$ & 0.07 & $\mathrm{~F}$ & 0.0076 & 0.261 & 20.5 \\
& NMSC & 8 & 1 & $0.85-1.17$ & 0.993 & $\mathrm{~F}$ & 0.0015 & 0.41 & 2.6 \\
& BCC & 7 & 1.01 & $0.85-1.21$ & 0.874 & $\mathrm{~F}$ & 0.0109 & 0.32 & 14.4 \\
\hline
\end{tabular}

NMSC, nonmelanoma skin cancer; BCC, basal cell carcinoma; SCC, squamous cell carcinoma; N, number of studies; F, fixed effects model; R, random effects model; $\mathrm{P}^{\mathrm{a}}$, test for association; $\mathrm{P}^{\mathrm{b}}$, test for heterogeneity

Table 4. Meta-Analysis of the XPD Asp312Asn Polymorphism with Risk of Skin Cancer

\begin{tabular}{llrlllllll}
\hline Comparison & Tumor type & $\mathrm{N}$ & $\mathrm{OR}$ & $95 \% \mathrm{CI}$ & $P^{a}$ & Model & $\chi^{2}$ & $P^{b}$ & $\mathrm{I}^{2}$ \\
\hline Asp/Asn $v$ s Asp/Asp & Overall & 10 & 1.04 & $0.96-1.14$ & 0.341 & $\mathrm{~F}$ & 0.0015 & 0.38 & 6.7 \\
& Melanoma & 6 & 1.06 & $0.96-1.17$ & 0.249 & $\mathrm{~F}$ & 0 & 0.599 & 0 \\
& NMSC & 4 & 1 & $0.85-1.17$ & 0.959 & $\mathrm{~F}$ & 0.025 & 0.136 & 45.9 \\
& BCC & 3 & 1.08 & $0.89-1.32$ & 0.416 & $\mathrm{~F}$ & 0.021 & 0.202 & 37.5 \\
Asn/Asn $v$ s Asp/Asp & Overall & 10 & 0.99 & $0.82-1.19$ & 0.888 & $\mathrm{R}$ & 0.0395 & 0.05 & 46.7 \\
& Melanoma & 6 & 1.16 & $1.00-1.35$ & 0.05 & $\mathrm{~F}$ & 0.0283 & 0.135 & 40.6 \\
& NMSC & 4 & 0.8 & $0.64-1.01$ & 0.065 & $\mathrm{~F}$ & 0 & 0.721 & 0 \\
dominant model & BCC & 3 & 0.8 & $0.60-1.05$ & 0.112 & $\mathrm{~F}$ & 0 & 0.516 & 0 \\
& Overall & 10 & 1.04 & $0.96-1.13$ & 0.313 & $\mathrm{~F}$ & 0.0022 & 0.285 & 16.7 \\
& Melanoma & 6 & 1.08 & $0.98-1.19$ & 0.1 & $\mathrm{~F}$ & 0 & 0.688 & 0 \\
recessive model & NMSC & 4 & 0.95 & $0.81-1.10$ & 0.471 & $\mathrm{~F}$ & 0.0151 & 0.192 & 36.8 \\
& BCC & 3 & 1.01 & $0.84-1.21$ & 0.939 & $\mathrm{~F}$ & 0.0199 & 0.193 & 39.2 \\
& Overall & 10 & 0.96 & $0.80-1.16$ & 0.689 & $\mathrm{R}$ & 0.0442 & 0.024 & 52.9 \\
& Melanoma & 6 & 1.06 & $0.84-1.34$ & 0.617 & $\mathrm{R}$ & 0.0417 & 0.056 & 53.6 \\
& NMSC & 4 & 0.81 & $0.65-1.00$ & 0.051 & $\mathrm{~F}$ & 0 & 0.7 & 0 \\
& BCC & 3 & 0.77 & $0.59-1.00$ & 0.046 & $\mathrm{~F}$ & 0 & 0.609 & 0 \\
\hline
\end{tabular}

NMSC, nonmelanoma skin cancer; BCC, basal cell carcinoma; SCC, squamous cell carcinoma; N, number of studies; F, fixed effects model; R, random effects model; $\mathrm{P}^{\mathrm{a}}$, test for association; $\mathrm{P}^{\mathrm{b}}$, test for heterogeneity

vs Lys/Lys: OR=1.01, 95\%CI=0.94-1.09, $p=0.710 ; \mathrm{Gln} /$ Gln $v s$ Lys/Lys: $\mathrm{OR}=1.08,95 \% \mathrm{CI}=0.98-1.19, p=0.133$; dominant model: $\mathrm{OR}=1.00,95 \% \mathrm{CI}=0.92-1.10, p=0.957$; recessive model: $\mathrm{OR}=1.07,95 \% \mathrm{CI}=0.98-1.17, p=0.134)$. In stratified analysis by tumor type, the present metaanalysis revealed that Lys751Gln polymorphism was not associated with nonmelanoma skin cancer risk, but contributed to risk of cutaneous melanoma $(\mathrm{Gln} / \mathrm{Gln} v s$ Lys/Lys: OR=1.15,95\%CI=1.02-1.29, $p=0.023$, Figure 2; dominant model: $\mathrm{OR}=1.09,95 \% \mathrm{CI}=1.01-1.18, p=0.036$, Figure 3 ). In further subgroup analysis by subtype of nonmelanoma, the results showed insignificant association of Lys751Gln polymorphism with basal cell carcinoma risk.

For XPD Asp312Asn polymorphism, the detailed results were listed in Table 4 . Significant between-study heterogeneity was seen in codominant model (Asn/Asn vs Asp/Asp: $\left.\mathrm{I}^{2}=46.7, p=0.050\right)$ and recessive model $\left(\mathrm{I}^{2}=52.9\right.$, $p=0.024)$, so the random-effects model was selected. There was no significant heterogeneity in the comparison of other genetic models and the fixed-effects model was used. In overall, the results indicated a nonsignificant association between the polymorphism and skin cancer (Asp/Asn $v s$ Asp/Asp: OR=1.04, 95\%CI=0.96-1.14, $p=0.341 ; \mathrm{Asn} /$ Asn vs Asp/Asp: $\mathrm{OR}=0.99,95 \% \mathrm{CI}=0.82-1.19, p=0.888$; dominant model: $\mathrm{OR}=1.04,95 \% \mathrm{CI}=0.96-1.13, p=0.313$; recessive model: $\mathrm{OR}=0.96,95 \% \mathrm{CI}=0.80-1.16, p=0.689)$. In subgroup analysis, we found that the Asp312Asn polymorphism was not linked to increased risk of cutaneous melanoma, nonmelanoma skin cancer or basal cell carcinoma.

\section{Sensitivity analysis}

Sensitivity analyses were performed after the sequential removal of each eligible study to assess the influence of each individual study on the pooled OR. The result of sensitivity analyses showed that any single study could not change the pooled ORs qualitatively, indicating robustness and reliability of our results.

\section{Publication bias}

Begg's funnel plots were created to evaluate the potential publication bias. The shapes of Begg's funnel plots did not reveal any evidence of obvious asymmetry 


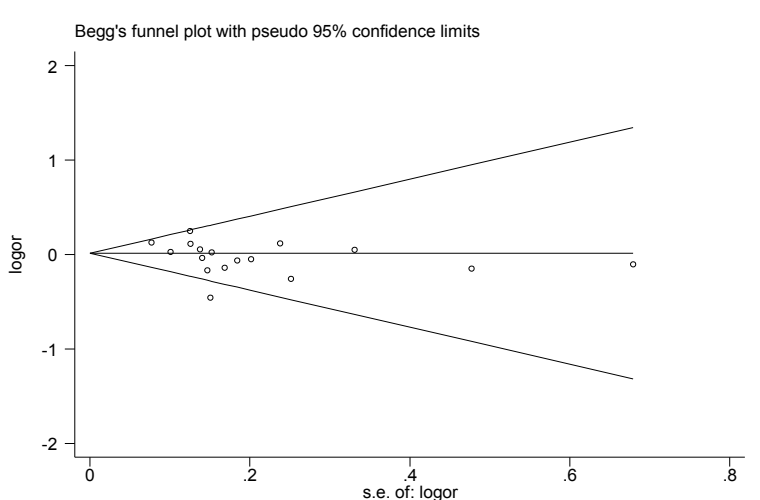

Figure 4. Begg's Funnel Plot of XPD Lys751Gln Polymorphism with Skin Cancer Risk ( Lys/Gln vs Lys/Lys)

in both XPD Lys751Gln and Asp312Asn polymorphisms (Figure 4). Concerning Lys751Gln polymorphism, the results of Begg's test suggested the absence of publication bias (Lys/Gln vs Lys/Lys: $p=0.174$; Gln/Gln vs Lys/Lys: $p=0.127$; dominant model: $p=0.064$; recessive model: $p=0.387)$. Similarly, no significant publication bias was demonstrated regarding Asp312Asn polymorphism (Asp/ Asn $v s$ Asp/Asp: $p=0.858$; Asn/Asn vs Asp/Asp: $p=0.592$; dominant model: $p=0.721$; recessive model: $p=0.283$ ).

\section{Discussion}

The damages of DNA resulted from various environmental factors, such as radiation, diet and smoking. Unrepaired DNA gives rise to gene mutations, chromosomal alterations, and genomic instability, which may activate carcinogenesis finally. DNA repair pathways play a crucial part in the removal of damages, repair of base alterations caused by UVR, recombination of homologous or nonhomologous end joining, and other injuries caused by many carcinogenic agents (Yu et al., 1999; Wood et al., 2001). The NER pathway is one of the most important DNA repair pathways, which removes bulky adducts, such as those caused by environmental agents, UVR induced DNA damage, crosslinks and oxidative damage (Sancar et al., 1993; Weeda et al., 1993).

XPD gene, located at chromosome 19q13.3, comprises 23 exons and spans about 54, 000 base pairs (Lamerdin et al., 1996). It encodes an evolutionarily conserved ATPdependent DNA helicase protein, a basal transcription initiation factor complex TFIIH, which is essential for NER (Weber et al., 1988), basal transcription, and apoptosis (Wang et al., 1996). The XPD gene is involved in the DNA helix opening, and is capable of removing helix-distorting base lesions produced by ultraviolet light and chemical agents (Lehmann et al., 2001). The XPD $751 \mathrm{Gln}$ and 312 Asn variants substantially alter the aminoacid electronic configuration in a domain important for the interaction with the helicase activator $\mathrm{p} 44$ and may change the function of XPD (Coin et al., 1998). Benhamou $S$ et al. have demonstrated that XPD Lys751Gln and Asp312Asn polymorphisms were associated with lower DNA repair capacity and a higher level of DNA adducts, which might be susceptible to cancer (Benhamou et al., 2002).
There are increasing interests in exploring the associations between the XPD Lys751Gln and/or Asp312Asn polymorphisms and susceptibility or resistance to cancer development. However, results remain conflicting rather than conclusive, which impelled researchers to pay attention to these two polymorphisms at a meta-analytical level. On the whole, three newly updated meta-analyses revealed that there were no evidence supporting that XPD Lys751Gln polymorphism contributed to prostate cancer (Ma et al., 2013), colorectal cancer (Zhang et al., 2011), or gastric cancer (Yin et al., 2013). On the contrary, other large sample meta-analyses proposed a greater risk in hepatocellular carcinoma (Guo et al., 2012), breast cancer (Yan et al., 2014), lung cancer (Li et al., 2014), glioma (Chen et al., 2012), and acute myeloid leukemia (AML) (Liu et al., 2014). For XPD Asp312Asn polymorphism, three meta-analyses proposed a greater risk in prostate cancer (Ma et al., 2013), gastric cancer (Yin et al., 2013), and esophageal cancer (Duan et al., 2012). However, other large sample meta-analyses showed that it did not contribute to breast cancer (Yan et al., 2014), lung cancer (Li et al., 2014), colorectal cancer (Zhang et al., 2011) or head and neck cancer (Hu et al., 2012) risk.

In reference to skin cancer susceptibility, the first study considering XPD Lys751Gln polymorphism as a potential molecular marker was performed by Dybdahl et al. in 1999 (Dybdahl et al., 1999), which investigated 40 cases and 40 controls. It showed that subjects carrying two A alleles (AA genotype) were at 4.3-fold higher risk of $\mathrm{BCC}$ than subjects with two $\mathrm{C}$ alleles, suggesting the variant $\mathrm{C}$-allele may be protective. Nonetheless, Winsey et al. (Winsey et al., 2000) reported that XPD Lys751Gln polymorphism was not related to the risk of cutaneous melanoma. Winsey et al. (Winsey et al., 2000) firstly reported the association between Asp312Asn polymorphism and skin cancer risk, and also, Asp312Asn polymorphism was not linked to susceptibility to cutaneous melanoma. Until now, a large amount of studies have been published to investigate the associations of XPD Lys751Gln or Asp312Asn polymorphisms with skin cancer susceptibility. Nevertheless, the results are poorly understood.

To further clarify the relationship between XPD Lys751Gln and/or Asp312Asn polymorphisms and skin cancer risk, we performed this meta-analysis. Our results suggested that XPD Lys751Gln polymorphism was not linked to increased risk of skin cancer. Subgroup analysis based on tumor type indicated the XPD Lys751Gln polymorphism was not associated with increased risk of nonmelanoma skin cancer, but significantly associated with increased risk of cutaneous melanoma (Gln/Gln vs. Lys/Lys: $\mathrm{OR}=1.15,95 \% \mathrm{CI}=1.02-1.29, \mathrm{P}=0.023$; dominant model: $\mathrm{OR}=1.09,95 \% \mathrm{CI}=1.01-1.18, \mathrm{P}=0.036$ ). Nevertheless, the pooled ORs indicated that Asp312Asn polymorphism was associated with neither overall nor stratified skin cancer in all genetic models.

When conducting meta-analysis, heterogeneity is one of the important issues. Although we minimized the likelihood by performing a careful search for published studies, using the explicit criteria for study inclusion, 
statistical significant heterogeneity still existed in three comparisons (dominant model for Lys751Gln polymorphism: I2=34.6, $\mathrm{P}=0.080 ;$ Asn/Asn vs. Asp/Asp for Asp312Asn polymorphism: $\mathrm{I} 2=46.7, \mathrm{P}=0.050$; and recessive model for Asp312Asn polymorphism: $\mathrm{I} 2=52.9$, $\mathrm{P}=0.024$ ). After subgroup analysis by tumor type, the heterogeneity was effectively decreased or removed, suggesting that certain effects of genetic variants are tumor type-specific. The results of sensitivity analyses showed no single study exhibited excessive influence, suggesting that the results of this meta-analysis were relatively stable. In addition, Begg's funnel plots and Begg's tests did not detect any publication bias and our results were unbiased.

Several limitations of this meta-analysis should be acknowledged when considering its contributions. First of all, the sample size of squamous cell carcinoma was relatively small. Thus, we do not have enough data to confirm the relationship between XPD polymorphisms with squamous cell carcinoma risk. Secondly, our metaanalysis's results were only applicable to Caucasians, and there was no relevant study from Asians or Africans. Hence, to evaluate the effect of XPD Lys751Gln and Asp312Asn polymorphisms on skin cancer risk, additional studies involving different ethnicities are needed. Thirdly, subgroup analyses based on environmental exposure were not performed on account of the insufficient data. Finally, gene-environment interactions were also not explored in this meta-analysis.

In summary, our meta-analysis suggests that XPD Lys751Gln polymorphism may contribute to the risk of cutaneous melanoma from currently available evidence. Considering the limited sample size and ethnicities included in the meta-analysis, larger and well-designed studies are required to confirm these findings. Moreover, future studies should evaluate gene-environment interactions to identify the role of the XPD polymorphisms in skin cancer.

\section{Acknowledgements}

This study was supported by a grant from the National Natural Science Foundation of China (No. 81271729).

\section{References}

Armstrong BK, Kricker A (2001). The epidemiology of UV induced skin cancer. J Photochem Photobiol B, 63, 8-18.

Begg CB, Mazumdar M (1994). Operating characteristics of a rank correlation test for publication bias. Biometrics, 50, 1088-101.

Baccarelli A, Calista D, Minghetti P, et al (2004). XPD gene polymorphism and host characteristics in the association with cutaneous malignant melanoma risk. Br J Cancer, $\mathbf{9 0 ,}$ 497-502.

Benhamou S, Sarasin A (2002). ERCC2/XPD gene polymorphisms and cancer risk. Mutagenesis, 17, 463-9.

Capell BC, Tlougan BE, Or low SJ (2009). From the rarest to the most common: insights from progeroid syndromes into skin cancer and aging. J Invest Dermatol, 129, 2340-50.

Coin F, Marinoni JC, Rodolfo C, et al (1998). Mutations in the XPD helicase gene result in XP and TTD phenotypes, preventing interaction between XPD and the p44 subunit of TFIIH. Nat Genet, 20, 184-8.
Chen DQ, Yao DX, Zhao HY, et al (2012). DNA repair gene ERCC1 and XPD polymorphisms predict glioma susceptibility and prognosis. Asian Pac J Cancer Prev, 13, 2791-4.

DerSimonian R, Laird N (1986). Meta-analysis in clinical trials. Control Clin Trials, 7, 177-88.

Dybdahl M, Vogel U, Frentz G, et al (1999). Polymorphisms in the DNA repair gene XPD: correlations with risk and age at onset of basal cell carcinoma. Cancer Epidemiol Biomarkers Prev, 8, 77-81.

Debniak T, Scott RJ, Huzarski T, et al (2006). XPD common variants and their association with melanoma and breast cancer risk. Breast Cancer Res Treat, 98, 209-15.

Duan XL, Gong H, Zeng XT, et al (2012). Association between XPD Asp312Asn polymorphism and esophageal cancer susceptibility: a meta-analysis. Asian Pac J Cancer Prev, 13, 3299-303.

Festa F, Kumar R, Sanyal S, et al (2005). Basal cell carcinoma and variants in genes coding for immune response, DNA repair, folate and iron metabolism. Mutat Res, 574, 105-11.

Guo LY, Jin XP, Niu W, et al (2012). Association of XPD and XRCC1 genetic polymorphisms with hepatocellular carcinoma risk. Asian Pac J Cancer Prev, 13, 4423-6.

Hu YY, Yuan H, Jiang GB, et al (2012). Associations between XPD Asp312Asn polymorphism and risk of head and neck cancer: a meta-analysis based on 7, 122 subjects. PLoS One, 7, 35220 .

Han J, Colditz GA, Liu JS, et al (2005). Genetic variation in XPD, sun exposure, and risk of skin cancer. Cancer Epidemiol Biomarkers Prev, 14, 1539-44.

Ji G, Lin Y, Cao SY, et al (2012). XPC 939A >C and 499C > T polymorphisms and skin cancer risk: a meta-analysis. Asian Pac J Cancer Prev, 13, 4983-8.

Kertat K, Rosdahl I, Sun XF, et al (2008). The Gln/Gln genotype of XPD codon 751 as a genetic marker for melanoma risk and Lys/Gln as an important predictor for melanoma progression: A case control study in the Swedish population. Oncol Rep, 20, 179-83.

Leiter U, Garbe C (2008). Epidemiology of melanoma and nonmelanoma skin cancer-the role of sunlight. $A d v \operatorname{Exp}$ Med Biol, 624, 89-103.

Lin JS, Eder M, Weinmann S (2011). Behavioral counseling to prevent skin cancer: a systematic review for the U.S. Preventive Services Task Force. Ann Intern Med, 154, 190-201.

Lovatt T, Alldersea J, Lear JT, et al (2005). Polymorphism in the nuclear excision repair gene ERCC2/XPD: association between an exon 6-exon 10 haplotype and susceptibility to cutaneous basal cell carcinoma. Hum Mutat, 25, 353-9.

Li C, Hu Z, Liu Z, et al (2006). Polymorphisms in the DNA repair genes XPC, XPD, and XPG and risk of cutaneous melanoma: a case-control analysis. Cancer Epidemiol Biomarkers Prev, 15, 2526-32.

Lamerdin JE, Stilwagen SA, Ramirez MH, et al (1996). Sequence analysis of the ERCC2 gene regions in human, mouse, and hamster reveals three linked genes. Genomics, 34, 399-409.

Lehmann AR (2001). The xeroderma pigmentosum group D (XPD) gene: one gene, two functions, three diseases. Genes Dev, 15, 15-23.

Li W, Li K, Zhao L, et al (2014). DNA repair pathway genes and lung cancer susceptibility: a meta-analysis. Gene, $\mathbf{5 3 8}$, $361-5$.

Liu D, Wu D, Li H, et al (2014). The effect of XPD/ERCC2 Lys751Gln polymorphism on acute leukemia risk: A systematic review and meta-analysis. Gene, 538, 209-16.

Mantel N, Haenszel W (1959). Statistical aspects of the analysis of data from retrospective studies of disease. J Natl Cancer 
Inst, 22, 719-48.

Millikan RC, Hummer A, Begg C, et al (2006). Polymorphisms in nucleotide excision repair genes and risk of multiple primary melanoma: the Genes Environment and Melanoma Study. Carcinogenesis, 27, 610-8.

Ma Q, Qi C, Tie C, et al (2013). Genetic polymorphisms of xeroderma pigmentosum group D gene Asp312Asn and Lys751Gln and susceptibility to prostate cancer: A systematic review and meta-analysis. Gene, 530, 309-14.

Pabalan N, Francisco-Pabalan O, Sung L, et al (2010). Metaanalysis of two ERCC2 (XPD) polymorphisms, asp312asn and lys751gln, in breast cancer. Breast Cancer Res Treat, 124, 531-41.

Povey JE, Darakhshan F, Robertson K, et al (2007). DNA repair gene polymorphisms and genetic predisposition to cutaneous melanoma. Carcinogenesis, 28, 1087-93.

Paszkowska-Szczur K, Scott RJ, Serrano-Fernandez P, et al (2013). Xeroderma pigmentosum genes and melanoma risk. Int J Cancer, 133, 1094-100.

Rubin AI, Chen EH, Ratner D (2005). Basal-cell carcinoma. $N$ Engl J Med, 353, 2262-9.

Siegel R, Naishadham D, Jemal A (2012). Cancer statistics, 2012. CA Cancer J Clin, 62, 10-29.

Sancar A, Tang MS (1993). Nucleotide excision repair. Photochem. Photobiol, 57, 905-21.

Shen MR, Jones IM, Mohrenweiser H (1998). Nonconservative amino acid substitution variants exist at polymorphic frequency in DNA repair genes in healthy humans. Cancer Res, 58, 604-8.

Salanti G, Amountza G, Ntzani EE, et al (2005). Hardy-Weinberg equilibrium in genetic association studies: an empirical evaluation of reporting, deviations, and power. Eur J Hum Genet, 13, 840-8.

Thompson JF, Scolyer RA, Kefford RF (2005). Cutaneous melanoma. Lancet, 365, 687-701.

Thirumaran RK, Bermejo JL, Rudnai P, et al (2005). Single nucleotide polymorphisms in DNA repair genes and basal cell carcinoma of skin. Carcinogenesis, 27, 1676-81.

Vogel U, Hedayati M, Dybdahl M, et al (2001). Polymorphisms of the DNA repair gene XPD: correlations with risk of basal cell carcinoma revisited. Carcinogenesis, 22, 899-904.

Weeda G, Hoeijmakers JH (1993). Genetic analysis of nucleotide excision repair inmammalian cells. Semin Cancer Biol, $\mathbf{4}$, 105-17.

Winsey SL, Haldar NA, Marsh HP, et al (2000). A variant within the DNA repair gene $\mathrm{XRCC} 3$ is associated with the development of melanoma skin cancer. Cancer Res, 60 , 5612-6.

Wood RD, Mitchell M, Sgouros J, et al (2001). Human DNA repair genes. Science, 291, 1284-9.

Weber CA, Salazar EP, Stewart SA, et al (1988). Molecular cloning and biological characterization of a human gene, ERCC2, that corrects the nucleotide excision repair defect in CHO UV5 cells. Mol Cell Biol, 8, 1137-46.

Wang XW, Vermeulen W, Coursen JD, et al (1996). The XPB and XPD DNA helicases are components of the p53-mediated apoptosis pathway. Genes Dev, 10, 1219-32.

Yin QH1, Liu C, Hu JB, et al (2013). XPD Lys751Gln and Asp312Asn polymorphisms and gastric cancer susceptibility: a meta-analysis of case-control studies. Asian Pac J Cancer Prev, 14, 231-6.

Yin J, Vogel U, Gerdes LU, et al (2003). Twelve single nucleotide polymorphisms on chromosome 19q13.2-13.3: linkage disequilibria and associations with basal cell carcinoma in Danish psoriatic patients. Biochem Genet, 41, 27-37.

Yu Z, Chen J, Ford BN, et al (1999). Human DNA repair systems: an overview. Environ Mol Mutagen, 33, 3-20.
Yan Y, Liang H, Light M, et al (2014). XPD Asp312Asn and Lys751Gln polymorphisms and breast cancer susceptibility: A meta-analysis. Tumour Biol, 35, 1907-15.

Zhang Y, Ding D, Wang X, et al (2011). Lack of association between XPD Lys751Gln and Asp312Asn polymorphisms and colorectal cancer risk: a meta-analysis of case-control studies. Int J Colorectal Dis, 26, 1257-64. 